\title{
Allocating healthcare resources-seriously ill people should have priority
}

We are sorry that the sense of the letter "Allocating healthcare resources-seriously ill people should have priority" (BMJ 2018;361:k1817, doi:10.1136/bmj.k1817) by Malcolm Segall was lost in abridging it as Letter of the Week in the print issue of 5 May 2018. The full letter with a link to the original rapid response is available here: https://www.bmj.com/content/361/ bmj.k1817. 